\title{
Determination of pore size distribution profile along wellbore: using repeat formation tester
}

\author{
Neda Nourzadeh $^{1} \cdot$ Seyed Reza Shadizadeh ${ }^{1} \cdot$ Abbas Khaksar Manshad $^{1}$
}

Received: 18 June 2016/ Accepted: 19 December 2016/Published online: 5 January 2017

(C) The Author(s) 2017. This article is published with open access at Springerlink.com

\begin{abstract}
Permeability and porosity are essential properties of hydrocarbon reservoirs that are series to compute the production rates and the original hydrocarbon in-place. Permeability and porosity are often specified by the pore size distribution. Knowing pore size distribution along wellbore can greatly help us in determining perforating depth, mud, and cement type, etc. There are several experimental methods for determining pore size distribution such as capillary pressure measurements, but these methods are time and cost consuming. The goal of this study is to analyze repeat formation tester (RFT) data to determine pore size distribution profile along wellbore. By analyzing RFT data, reservoir pressure, fluids density, fluids contact, and capillary pressure were determined. Pore radiuses were calculated from capillary pressure, and pore size distribution was determined from frequency analysis of pore radius. On the other hand, mercury injection experiments were carried out on several core samples, and the results were used to determine pore size distribution from core analysis. At the last step, the pore size distribution obtained from RFT and core analyses was compared. Result of comparison shows acceptable accuracy of RFT pore size distribution.
\end{abstract}

Keywords Pore size distribution · Capillary pressure . RFT · Fluids density · Fluids contact

Seyed Reza Shadizadeh

shadizadeh@put.ac.ir

1 Department of Petroleum Engineering, Abadan Faculty of Petroleum Engineering, Petroleum University of Technology, Northern Bowarde, Abadan 6318714331, Iran

\author{
List of symbols \\ $g \quad$ Conversion factor \\ $h \quad$ Vertical depth (ft) \\ PSD Pore size distribution \\ $P_{\mathrm{c}} \quad$ Capillary pressure (psi) \\ RFT Repeat formation tester \\ $\rho_{\mathrm{o}} \quad$ Oil density $\left(\mathrm{gr} / \mathrm{cm}^{3}\right)$ \\ $\rho_{\mathrm{w}} \quad$ Water density $\left(\mathrm{gr} / \mathrm{cm}^{3}\right)$ \\ $\theta \quad$ Contact angle $\left(^{\circ}\right)$ \\ $\sigma \quad$ Interfacial tension (dyne/cm)
}

\section{Introduction}

The proper description of the pore size distribution (PSD) and the pore structure is significant, because the nature of the pores is highly impressed the mass transfer through the grains (Bacskay et al. 2014). A quantitative characterization of the range of pore sizes in a sample is provided by the pore size distribution (Giesche 2006). Pore sizes are divided into three clusters: (i) micropores, having radius $(<1 \mathrm{~nm})$; (ii) mesopores, having radius between 1 and $25 \mathrm{~nm}$; (iii) macropores, having radius ( $>25 \mathrm{~nm}$ ) (Bacskay et al. 2014). One method from variety of experimental techniques is used to measure pore size distribution by depending on the range of the pore sizes that a porous material contains (Mourhatch et al. 2011). Dukhin et al. (2013) analyzed pore size and porosity of porous materials by using electroacoustic and high-frequency conductivity. Chalk et al. (2012) determined pore size distribution from challenge coreflood testing by colloidal flow. Martin et al. (2007) determined the pore-throat size distribution in plugs by using the centrifuge as a tool. Dong et al. (2007) exacted 
pore networks by using X-ray microtomography (micro$\mathrm{CT}$ ) to image rock cuttings of poorly consolidated. Elgaghah (2007) used scanning electron microscope (SEM) and determined the pore size distribution. Howard et al. (1993) used proton nuclear magnetic resonance (NMR) method and showed that the longitudinal relaxation time (T1) in water-saturated sandstones is related closely to the pore size distributions.

This paper presents determination of pore size distribution profile along wellbore using repeat formation tester (RFT) data.

\section{Methodology}

Figure 1 shows the flowchart of the procedure in this study. The steps of procedure have been explained below.

\section{Determination of formation pressure}

The formation pressure for each depth was obtained by plotting pressure versus time for each test. RFT profile was obtained by plotting formation pressure versus depth. From this profile, fluid density and fluid contacts were determined. A pressure gradient over the hydrocarbon-bearing interval of the reservoir and a pressure gradient over the water-bearing interval of the reservoir were specified by plotting pressure measurements. From these pressure

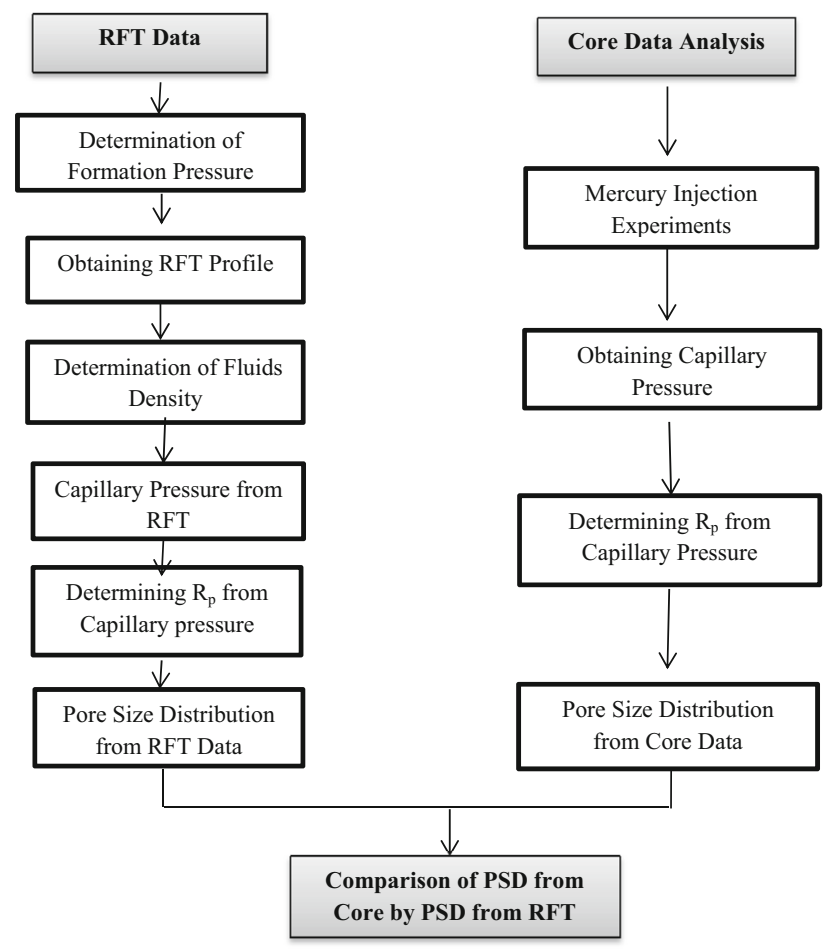

Fig. 1 Flowchart of the procedure of this study gradients, the fluid densities were calculated by using this equation:

$\rho=\frac{\Delta P / \Delta D}{0.4335}$

where $\rho$ is fluid density in $\mathrm{gr} / \mathrm{cm}^{3}$ and $\Delta P / \Delta D$ is the pressure change over the appropriate depth interval in $\mathrm{psi} / \mathrm{ft}$ (Raymer and Freeman 1984).

\section{Capillary pressure from RFT data}

Capillary pressure was determined by using the capillary pressure (Eq. 2) which depended on fluid densities, fluid pressures, and the elevation within a capillary system:

$P_{\mathrm{c}}=P_{\mathrm{h}}-P_{\mathrm{w}}=g h\left(\rho_{\mathrm{w}}-\rho_{\mathrm{h}}\right)$

where $P_{\mathrm{c}}$ is capillary pressure, $P_{\mathrm{h}}$ is hydrocarbon pressure at a given elevation, $P_{\mathrm{w}}$ is water pressure at a given elevation, $h$ is the elevation above the free water level (hydrocarbon-water contact), $g$ is acceleration due to gravity, $\rho_{\mathrm{w}}$ is water density, and $\rho_{\mathrm{h}}$ is hydrocarbon density (Raymer and Freeman 1984).

Rearranged, Eq. (2) becomes:

$P_{\mathrm{c}}=1.42 h\left(\rho_{\mathrm{w}}-\rho_{\mathrm{h}}\right)$

where $P_{\mathrm{c}}$ is capillary pressure in psi, $h$ is the elevation above the free water level in $\mathrm{m}, \rho_{\mathrm{h}}$ is hydrocarbon density in $\mathrm{gr} / \mathrm{cm}^{3}$, and $\rho_{\mathrm{w}}$ is water density in $\mathrm{gr} / \mathrm{cm}^{3}$ (Raymer and Freeman 1984).

\section{Determination of pore radius}

Pore radius was calculated through capillary pressure considerations. The correlation between capillary pressure and pore radius is:

$P_{\mathrm{c}}=\frac{2 \gamma \cos \theta}{r_{\mathrm{p}}}$

where $P_{\mathrm{c}}$ is capillary pressure, $\gamma$ is interfacial (surface) tension, $\theta$ is contact angle, and $r_{\mathrm{p}}$ is pore (capillary) radius. Holcott apperceived that adhesion tension (the product of cosine of contact angle and surface tension) was typically 30 dynes/cm in many oil-bearing reservoirs at reservoir conditions. Hough, Rzasa, and Wood apperceived that adhesion tension of gas-bearing reservoirs was 35 dynes/ $\mathrm{cm}$. By using these values, Eq. 4 is written as:

$r_{\mathrm{p}}=\frac{a}{p_{\mathrm{c}}}$

where $r_{\mathrm{p}}$ is pore radius in $\mu \mathrm{m}, P_{\mathrm{c}}$ is capillary pressure in psi, and $a$ is equal to 8.7 for oil-water at typical reservoir conditions (Raymer and Freeman 1984).

Pore radiuses were calculated from capillary pressure data. Pore radius and frequency of each pore radius were 


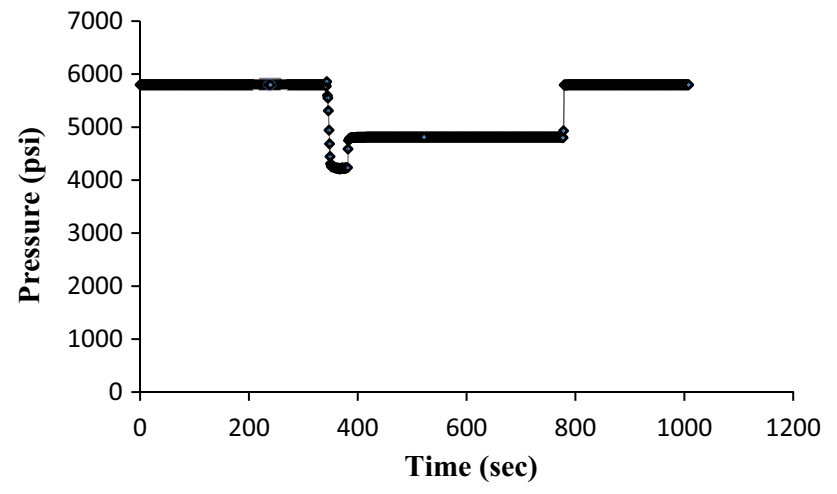

Fig. 2 Formation pressure for depth of $2858 \mathrm{~m}$

Table 1 Formation pressure versus depth of well Koushk No. A

\begin{tabular}{llll}
\hline Depth $(\mathrm{m})$ & Pressure (psi) & Depth $(\mathrm{m})$ & Pressure (psi) \\
\hline 2477.0 & 3976.7 & 3190.0 & 5230.4 \\
2850.3 & 4802 & 3199.0 & 5243.3 \\
2858.0 & 4811.6 & 3342.0 & 5437.7 \\
2958.0 & 4954.8 & 3375.0 & 5488.7 \\
2985.0 & 4972.1 & 3400.0 & 5527.6 \\
3005.0 & 4982.7 & 3641.0 & 5776.2 \\
3020.0 & 5038.5 & 3645.0 & 5780.8 \\
3027.5 & 5020.9 & 3650.0 & 5787.7 \\
3048.0 & 5025.6 & 3654.5 & 5794 \\
3064.0 & 5056.2 & 3659.0 & 5800.1 \\
3076.0 & 5057.2 & 3735.0 & 5967.2 \\
3085.0 & 5103.3 & 3750.0 & 5989.5 \\
3102.5 & 5105.5 & 3775.0 & 6028 \\
3119.7 & 5135.8 & 3982.0 & 6477 \\
3127.0 & 5130.1 & 3985 & 6469 \\
\hline
\end{tabular}

analyzed, and pore size distribution was plotted in four different intervals above water-oil contact.

Data of well Koushk No. A located in Yadavaran oilfield including RFT tests and core samples were used in this work. Mercury injection experiment was carried out on core samples, the results were used to determine pore size distribution, and comparison with the results obtained from the RFT data.

\section{Results and discussion}

Figure 2 shows formation pressure for depth of $2858 \mathrm{~m}$ of well Koushk No. A is 4811.6 psi. The results of formation pressure determination for 30 tests are represented in Table 1. Figure 3 shows formation pressures versus depths plot; different slope of the two lines indicates that fluid phase of the reservoir has been changed. Oil zone is in the region of the upper line, and the water zone is in the region

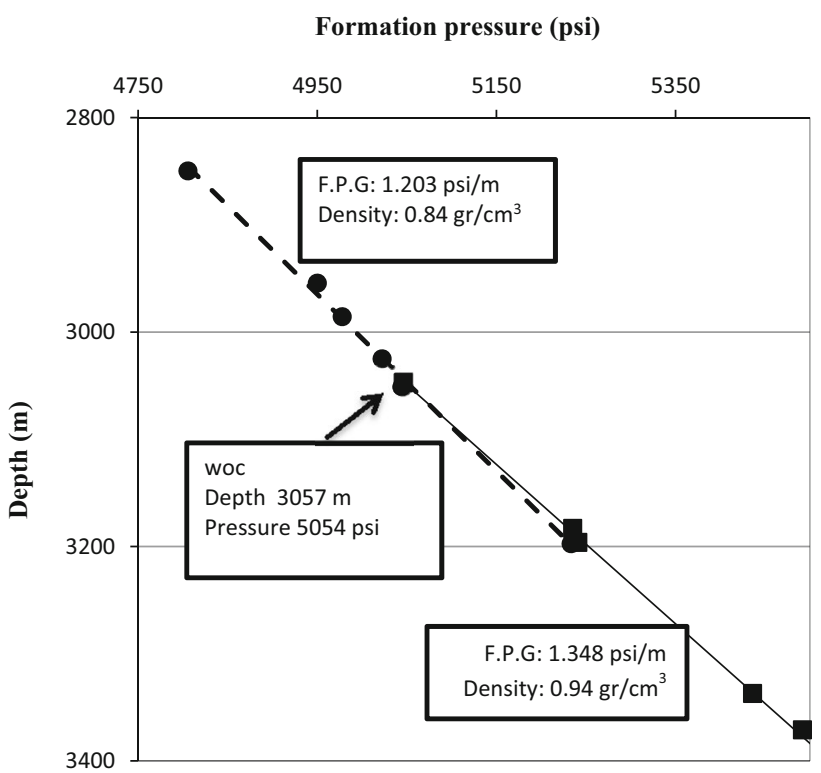

Fig. 3 Observed WOC in RFT data

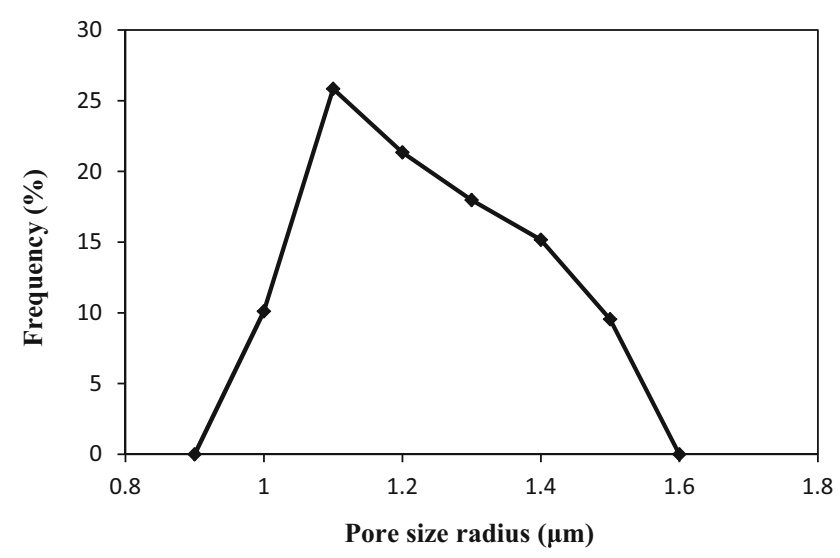

Fig. 4 Pore size distribution for interval of 2978-3005 m

Table 2 Examples of capillary pressure calculation

\begin{tabular}{lllll}
\hline $\begin{array}{l}\text { Depth } \\
(\mathrm{m})\end{array}$ & $\begin{array}{l}h=\text { Depth- } \\
\text { WOC }(\mathrm{m})\end{array}$ & $\begin{array}{l}\rho_{\mathrm{w}}=\frac{\Delta P / \Delta D}{0.4335} \\
\left(\mathrm{gr} / \mathrm{cm}^{3}\right)\end{array}$ & $\begin{array}{l}\rho_{\mathrm{h}}=\frac{\Delta P / \Delta D}{0.4335} \\
\left(\mathrm{gr} / \mathrm{cm}^{3}\right)\end{array}$ & $\begin{array}{l}P_{\mathrm{c}}=\frac{h\left(\rho_{\mathrm{w}}-\rho_{\mathrm{h}}\right)}{2.3} \\
(\mathrm{psi})\end{array}$ \\
\hline 2675.07 & 381.93 & 0.95 & 0.84 & 59.65 \\
3050.28 & 24.72 & 0.95 & 0.84 & 3.86 \\
\hline
\end{tabular}

of the lower line. The intersection of the two lines indicates water-oil contact at the depth of $3057 \mathrm{~m}$. The capillary pressure for every depth at the reservoir was determined by knowing the difference of the fluid densities from the previous section and by using Eq. (3). Calculations of capillary pressure for two depths are represented in Table 2 as examples.

Figure 4 shows the pore size distribution curve for interval 2978-3005 m of well Koushk No. A, which 


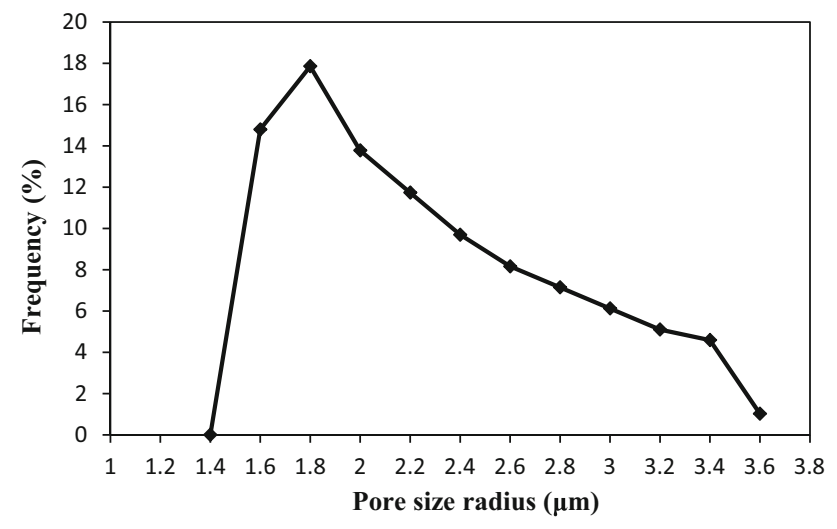

Fig. 5 Pore size distribution for interval 3005-3034 m

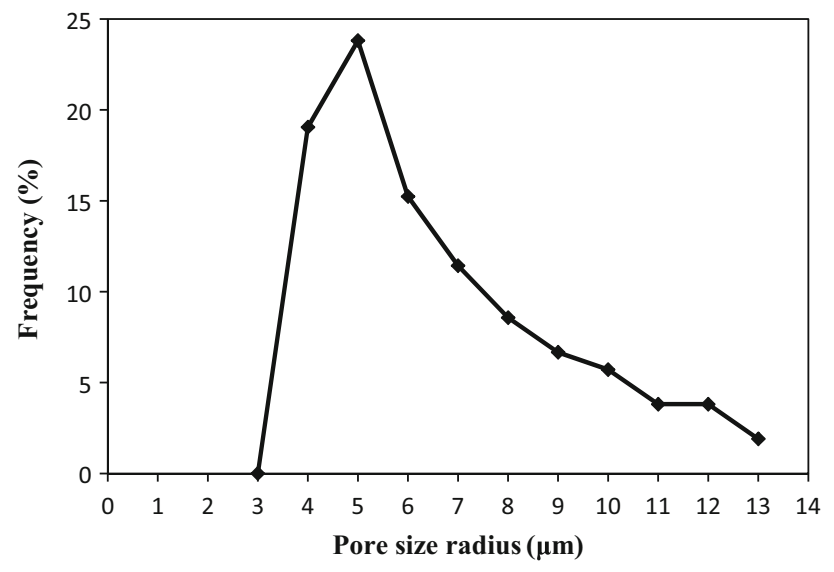

Fig. 6 Pore size distribution for interval 3035-3050 m

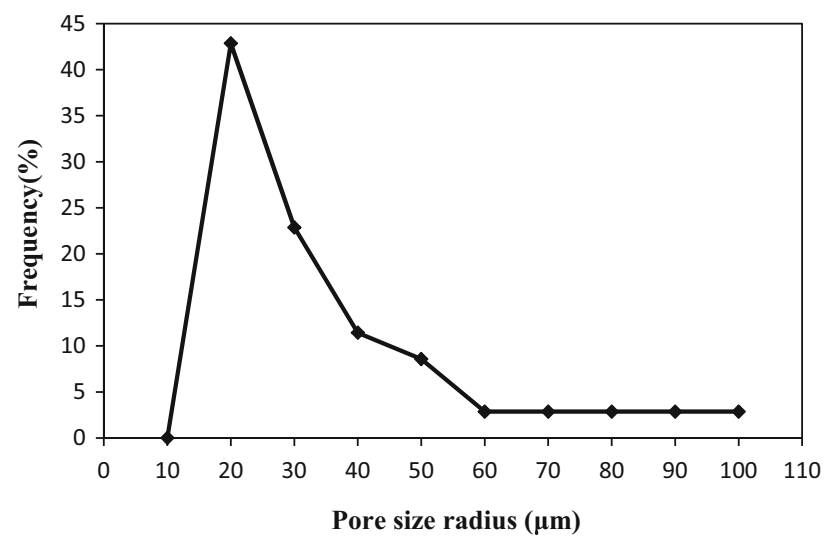

Fig. 7 Pore size distribution for interval 3051-3056 m

indicates radius of pores is between 0.9 and $1.5 \mu \mathrm{m}$. Figure 5 shows the pore size distribution curve for interval 3005-3034 m of well Koushk No. A. Pore size distribution diagram indicates radius of pores is between 1.4 and $3.6 \mu \mathrm{m}$, and the vertical axis of the diagram indicates the corresponding frequency of the pores. Figure 6 shows the pore size distribution curve for interval $3035-3050 \mathrm{~m}$ of

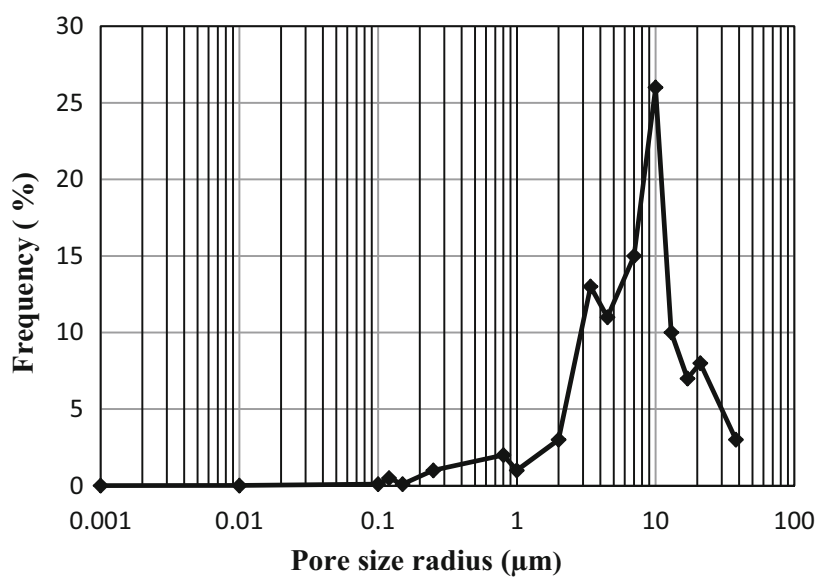

Fig. 8 Pore size distribution for depth $3038.5 \mathrm{~m}$

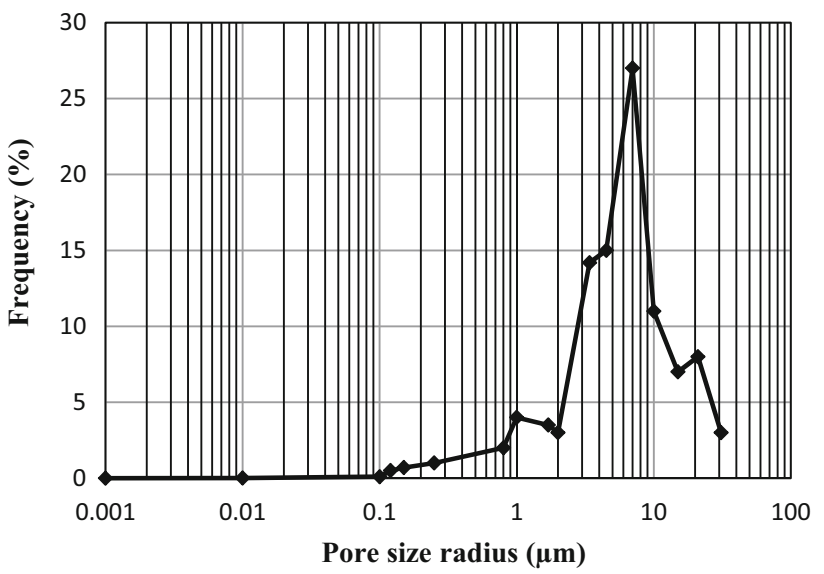

Fig. 9 Pore size distribution for depth $3040.8 \mathrm{~m}$

well Koushk No. A, which indicates radius of pores is between 3 and $13 \mu \mathrm{m}$. Figure 7 shows the pore size distribution curve for interval 3051-3056 m, which indicates radius of pores is between 10 and $100 \mu \mathrm{m}$.

Figure 8 shows the pore size distribution for depth $3038.5 \mathrm{~m}$. During mercury injection experiment, due to extremely high pressure the mercury penetrates into very small pores. The results of core analysis in this depth show that most of the pores (about $85 \%$ ) have a radius between 1 and $20 \mu \mathrm{m}$ and about $26 \%$ of the pores have a radius of $10 \mu \mathrm{m}$. Figure 9 shows the pore size distribution curve for depth $3040.8 \mathrm{~m}$. The curve shows that most of the pores have a radius between 2 and $15 \mu \mathrm{m}$ and about $27 \%$ of the pores have a radius of $7 \mu \mathrm{m}$. The PSD curve of this depth is very similar to the PSD curve of the depth $3038.5 \mathrm{~m}$, which is shown in Fig. 8 while the curve has a small shift to the left.

Figure 10 shows the pore size distribution for depth $3045.5 \mathrm{~m}$. About $80 \%$ of the pores have a radius between 1.7 and $13 \mu \mathrm{m}$, and $26 \%$ of the pores have a radius of $6 \mu \mathrm{m}$. Figure 11 shows the pore size distribution for depth 


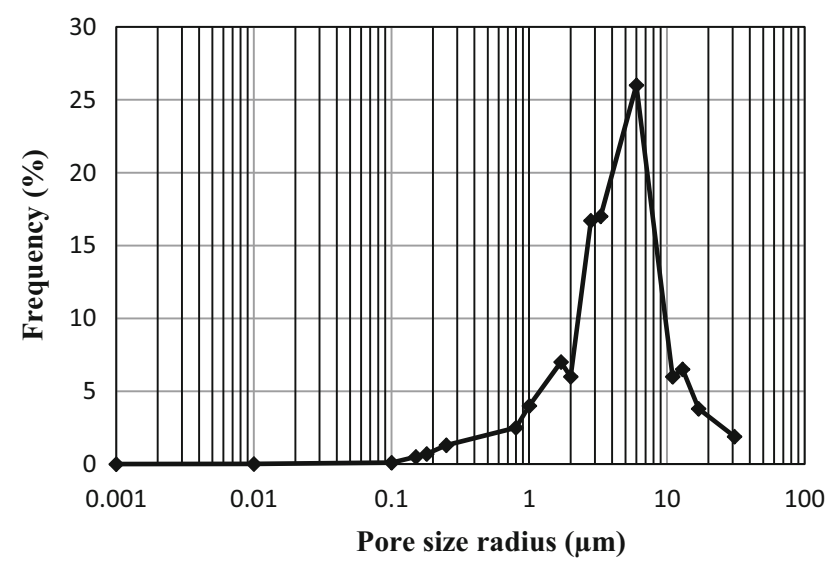

Fig. 10 Pore size distribution for depth $3045.5 \mathrm{~m}$

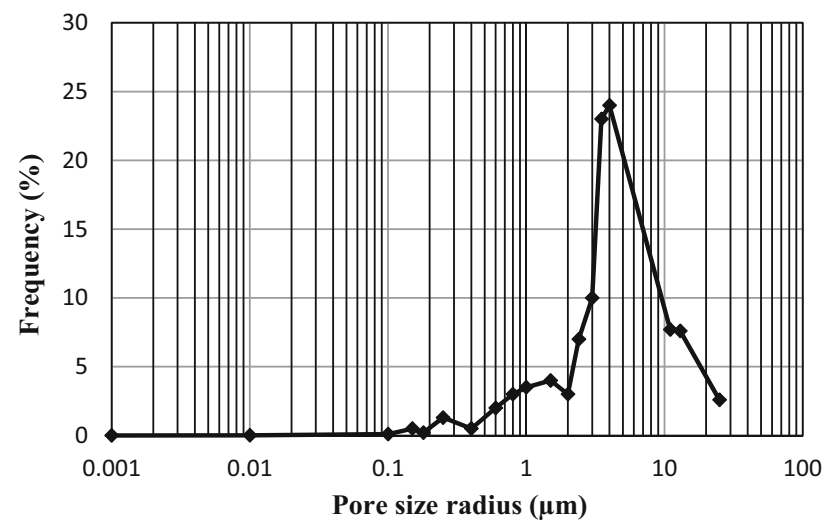

Fig. 11 Pore size distribution for depth $3048.9 \mathrm{~m}$

$3048.9 \mathrm{~m}$. About $75 \%$ of the pores have a radius between 1.5 and $13 \mu \mathrm{m}$, and $24 \%$ of the pores have a radius of $4 \mu \mathrm{m}$.

Pore size distributions were plotted for four core plugs in the interval of 3035-3050 m. These four curves are very similar and have negligible differences. The pore size distribution curve due to core plug of depth $3045.5 \mathrm{~m}$ was compared with the results obtained from RFT data in a similar interval. Figure 12 shows the result of comparison between RFT and core. The results illustrate that the main part of the curves for this interval is almost similar to a little differences where these differences may be occurred because the nature and scale of the tests are different.

Major section of the pore size distribution curve from both methods (core analysis and RFT analysis) has an acceptable correlation. Different scale of RFT test and mercury injection experiment causes some differences in the pore size distribution curves, for example, in the mercury injection test, due to high pressure, mercury may penetrate into small pores, while RFT test was performed at the reservoir condition (at reservoir pressure) and only large pores was determined.

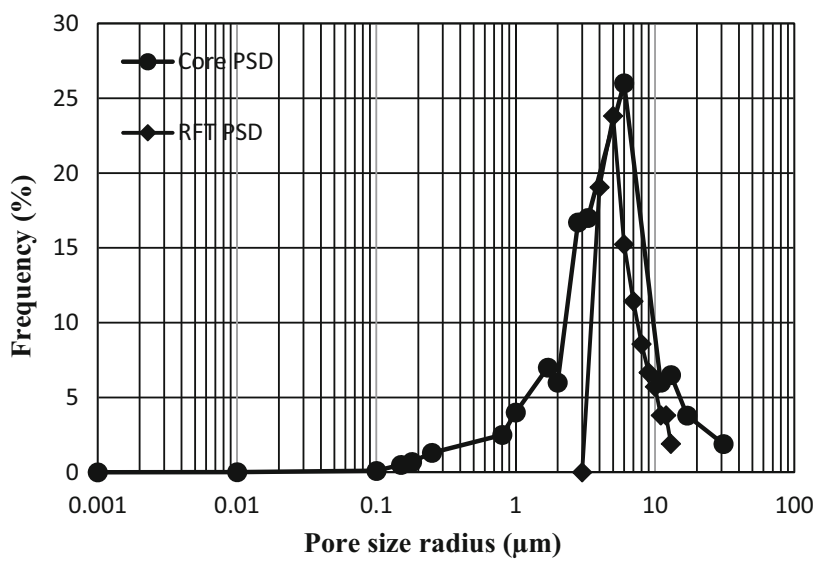

Fig. 12 Result of comparison between PSD from RFT and core

\section{Conclusions}

1. Several parameters include in capillary pressure $\left(P_{\mathrm{c}}\right)$, pore radius $\left(r_{\mathrm{p}}\right)$, fluids density, depth of water-oil contact (WOC), and at the last step pore size distribution were obtained by analyzing RFT data.

2. Pore size distribution was obtained from RFT data in four pay zones of the reservoir. The results of one zone compared with the pore size distribution which was derived from mercury injection experiment. The results of this comparison show acceptable correlation between RFT results and core results.

3. There are some differences between the results of RFT method and MICP method. The source of these differences is the difference in the nature of data and the scale of the tests. RFT is performed at reservoir condition, while MICP test is performed in the laboratory condition, and it is usual that their results have some differences.

4. Extremely high pressure enters the mercury into very small pores, and this method measures very small pores which have not important role in fluid flow in a reservoir, while RFT method measures the fluid pressure in the reservoir condition so it is concluded that the accuracy of MICP is very high but the results of RFT method are more realistic.

5. Laboratory methods of determining pore size distribution are very expensive and time consuming, while the method which used in this study is a fast and cheap method.

Acknowledgements The authors would like to thank the Petroleum University of Technology for supporting in this study.

Open Access This article is distributed under the terms of the Creative Commons Attribution 4.0 International License (http:// creativecommons.org/licenses/by/4.0/), which permits unrestricted use, distribution, and reproduction in any medium, provided you give 
appropriate credit to the original author(s) and the source, provide a link to the Creative Commons license, and indicate if changes were made.

\section{References}

Bacskay I, Sepsey A, Felinger A (2014) Determination of the pore size distribution of high-performance liquid chromatography stationary phases via inverse size exclusion chromatography. J Chromatogr A 1339:110-117

Chalk P, Gooding N, Hutten S, You Z, Bedrikovetsky P (2012) Pore size distribution from challenge coreflood testing by colloidal flow. Chem Eng Res Des 90:63-77

Dong H, Touati M, Blunt MJ (2007) Pore network modeling: analysis of pore size distribution of Arabian core samples. SPE paper 105156, SPE Middle East oil and gas show and conference, Manama, Bahrain, 11-14 March

Dukhin A, Swasey S, Thommes M (2013) A method for pore size and porosity analysis of porous materials using electroacoustics and high frequency conductivity. Colloids Surf A 437:127-132
Elgaghah S (2007) A novel technique for the determination of microscopic pore size distribution of heterogeneous reservoir rocks. SPE paper 107750, Asia pacific oil and gas conference, Jakarta, Indonesia, 30 October-1 November

Giesche H (2006) Mercury porosimetry: a general (practical) overview. Part Part Syst Charact 23:9-19

Howard JJ, Kenyon WE, Straley C (1993) Proton magnetic resonance and pore size variations in reservoir sandstones. SPE Form Eval 8:194-200

Martin CA, Ramia M, Barberis L (2007) The centrifuge as a tool to determine the pore-throat size distribution in plugs. SPE paper 107781, Latin American \& Caribbean petroleum engineering conference, Buenos Aires, Argentina, 15-18 April

Mourhatch R, Tsotsis TT, Sahimi M (2011) Determination of the true pore size distribution by flow permporometry experiments: an invasion percolation model. J Membr Sci 367:55-62

Raymer LL, Freeman PM (1984) In-situ determination of capillary pressure, pore throat size and distribution, and permeability from wireline data. I: SPWLA 25th annual logging symposium. Society of petrophysicists and well-log analysts, New Orleans, Louisiana, 10-13 June 